\title{
Dimensional Quality Oriented Reliability Modeling for Complex Manufacturing Processes
}

\author{
Faping Zhang, Jiping Lu*, Yan Yan, Shuiyuan Tang \\ School of Mechanical Engineering, Beijing Institute of Technology, 100081 Beijing, China \\ Chao Meng \\ Department of Mechanical Engineering, RWTH Aachen, 52062 Aachen, Germany \\ Received 19 August 2011 \\ Accepted 25 November 2011
}

\begin{abstract}
The stability of process is certainly one of the most important aspects to fulfill the task. Now, it is still a challenge to put forward a model to deal with the stability of manufacturing process mathematically. Aiming at solving the problem, this paper discusses the issues by the notion of manufacturing process reliability and its sensitivity. Based on state space model, the process performance function which is used to describe the relations between the key product characters (KPC) of the machined part and the key control characters (KCC) of multistage process in machining system has been built. Furthermore, the measure index to the process reliability of multistage machining system is proposed, which facilitates the forming of the ways to model the process reliability mathematically. To determine the weakest stage during machining process, the sensitivity of process reliability to KCC of the process has been put forward and the corresponding ways how to calculate it. Because of the multistage process is highly non-linear process, chaos optimization algorithm and mutative scale chaos optimization algorithm are used to calculate this kind of robust reliability index. Finally, A simple 2-D case study has been used to validate the proposed model. It shows that the process reliability can be calculated effectively.
\end{abstract}

Keywords: state space model, mutative chaos optimization algorithm, process reliability modeling, multistage machining process.

\section{Introduction}

Manufacturing process is the bridge to connect the design reliability and real product reliability. There are some quality instability phenomena due to the random variation in sources such as materials, machines, fixtures and machining methods in the process. Stability of any machining process is one of the most important key factors to measure process reliability. Zhang et al have showed that issues of quality and reliability count for 24 percent of the manufacturing processes in the industry [1]. Nowadays, the stability of machining process is not high which the requirement of certain industries such aviation industry due to the variation in the machining of products which involve complex machining process, special raw material and complex structure. Thus, this makes research on the machining process reliability one of the hottest research fields in modern machining research.

J.K. Jacob [2] presents the method to detect any change in intensity parameter and a control chart procedure is presented for process reliability monitoring. Mocko and Hines, JW [3-4] study the reliability prediction of equipments for process reliability under the special conditions. In order to evaluate the manufacturing process reliability of MEMS, M. Dardalhon [5] presented general set of test structures, associated test and analysis methods. After an intensive review of literatures on reliability and process variation, Alma [6] gave a broad perspective of "reliability and process variation aware design of integrated circuits”.

But those researchers are more concerned about the reliability of the machining system as the reliability of used machine and the fixtures, or the design of reliability [7-8]. Multiple stages machining process is one of

*Corresponding author: jipinglu@bit.edu.cn.

This project is financed by the National Department Fundamental Research Foundation of China (DEDP1901). 
the important manufacturing processes in modern manufacturing field. Some researchers have done considerable amount of work on the product quality and machining system reliability [9-11] but the task reliability of the machining process, normally how to keep the quality of the machined products stable and how to establish the mathematical model to describe machining process reliability is not sufficient. So, this seems to be a prospective area to conduct research.

Now-a-days, there is a phenomenon that most machining processes are unstable processes and process reliability is not high. There have been many factors contributing to this situation, such as the lack of clarity in the relations between the Key process characters and the reliability of the machined parts. So, it is an important task to study the mechanism of the forming and representation of the machining process reliability.

This paper deals machining process reliability with the exact definition of process reliability, modeling of machining process reliability and sensitive analysis. The remainder of the paper is organized as follows; section 2 gives the linear state modeling of process reliability and the corresponding sensitivity determination. In section 3 a simple two-dimensional example is used to illustrate the implementation of the newly proposed method in section 2. Finally, the conclusion of the paper is drawn in section 4.

\section{Modeling of Process Reliability}

\subsection{The Conception of Process Reliability}

Process reliability has been studied against the process defects as well as product reliability against failure. The reliability of advanced manufacturing system can be dealt as basic reliability and task reliability. Here, the reliability of machining process is referred as the ability of process to meet the design requirement, namely the task reliability. There are many defects which lead to such process failure as unexpected workpiece and fixture contact, improper workpiece orientation, unsuitable controlled dimension, unacceptable surface roughness, distorting, etc. According to the normal definition of the reliability and considering the special features of manufacturing system, the definition of process reliability is given as: "under the stated condition and stated time, the ability of the manufacturing system to ensure the process parameters; quality stably is the reliability of machining process - namely the ability of process to manufacture the quality bound product stability”. Process reliability consider the uncertainty of dimension, material and process parameters, and analyze how these random variables effect on the process reliability through numerical simulation and various resolution. The task of manufacturing system is to produce the quality bound part. So, quality parameter of produced part is one of the most important indexes to process reliability, which is used in this paper to formulate the model of machining process reliability.

\subsection{Performance Function of Machining Process}

The machined part of machining process is modeled as a vector by stacking up all part feature vectors, so we have

$$
\boldsymbol{X}^{L}(\boldsymbol{k})=\left(\begin{array}{llll}
\boldsymbol{X}_{\mathbf{1}}^{L}(\boldsymbol{k}) & \boldsymbol{X}_{2}^{L}(\boldsymbol{k}) & \cdots & \boldsymbol{X}_{n}^{L}(\boldsymbol{k})
\end{array}\right)
$$

where $X_{n}^{L}(k)$ denotes a part feature parameter. According to the coordinate system transformation theory, part feature representation can be transformed from coordinate system LCS to coordinate system GCS as follows

$$
\boldsymbol{X}^{G}(\boldsymbol{k})=\boldsymbol{T}_{L-G}(k) X^{L}(k)+R_{L}^{G}(k)
$$

where $\boldsymbol{T}_{\boldsymbol{L}-\mathrm{G}}(\boldsymbol{k})$ denotes the transformation matrix transforming vector representation in coordinate system LCS into the coordinate system GCS. $\boldsymbol{R}_{L}^{G}(\boldsymbol{k})$ denotes the representation of the original coordinate system LCS in coordinate system GCS. In the same way part feature representation can be transformed from coordinate system WCS to coordinate system LCS as follows:

$$
\boldsymbol{X}^{L}(\boldsymbol{k})=\boldsymbol{T}_{W-L}(\boldsymbol{k}) \boldsymbol{X}^{W}(\boldsymbol{k})+\boldsymbol{R}_{W}^{L}(\boldsymbol{k})
$$

where $\boldsymbol{T}_{W-L}(\boldsymbol{k})$ denotes the matrix that transforms vector representation in coordinate system WCS into the coordinate system LCS, while $\boldsymbol{R}_{W}^{L}(\boldsymbol{k})$ denotes the representation of the original of coordinate system WCS in coordinate system LCS.

Combining Eq. (1) and (2) we have

$$
\begin{aligned}
\boldsymbol{X}^{W}(\boldsymbol{k}) & =\boldsymbol{T}_{W-L}^{-1}(\boldsymbol{k}) \boldsymbol{T}_{L-G}^{-1}(\boldsymbol{k}) \boldsymbol{X}^{G}(\boldsymbol{k}) \\
& \quad-\boldsymbol{T}_{W-L}^{-1}(\boldsymbol{k}) \boldsymbol{T}_{L-G}^{-1}(\boldsymbol{k}) \boldsymbol{R}_{L}^{G}(\boldsymbol{k})-\boldsymbol{T}_{W-L}^{-1}(\boldsymbol{k}) \boldsymbol{R}_{W}^{L}(\boldsymbol{k})
\end{aligned}
$$

where $T_{\mathrm{W}-\mathrm{L}}^{-1}(k), T_{\mathrm{L}-\mathrm{G}}^{-1}(k)$ are inverse matrices of $T_{\mathrm{W}-\mathrm{L}}(k)$, $T_{\mathrm{L}-\mathrm{G}}(k)$ and denotes inverse transformation of vector representation from LCS to WCS and GCS to LCS respectively.

The above part feature positions are nominal value where no factual errors are considered. As many factors 
will cause the deviation of workpiece features, so the dimensional errors emerge which causes failure of the machining process. The emergence and stacking up of the dimensional errors can be described by Stream of Variation (SOV) model.

The performance function of process is used to describe the relations of key control characters (KCC) of the process and key product characters (KPC) of the machined part mathematically. In fact machining process can be reckoned as discrete time-varying system, while the machining stage is used as time index and the dimensional errors as outputs. In this condition, state space model can be used to construct performance function of process.

$$
\Delta X(\boldsymbol{k})=A(\boldsymbol{k}) \Delta X(\boldsymbol{k}-\mathbf{1})+B(\boldsymbol{k}) U(\boldsymbol{k})+\boldsymbol{W}(\boldsymbol{k})
$$

where $\Delta X(\boldsymbol{k})$ are the dimensional deviation vector of the $\mathrm{k}$ stage, which is one of the important parts of KPC, while $\Delta X(\boldsymbol{k}-\mathbf{1})$ is that of the k-1 stage. $A(\boldsymbol{k})$ is the dimensional deviation state matrix which reflects the influence of feature state vector at the k-1 stage on dimensional deviation vector of the $\mathrm{k}$ stage; $B(\boldsymbol{k})$ shows how errors $\Delta X(\boldsymbol{k})$ depend on the newly introduced machining errors $U(\boldsymbol{k})$, which is the one part of KCC. And vectors $\boldsymbol{W}(\boldsymbol{k})$ take into account the residuals after linearization and un-modeled effects.Fig.1 illustrates the multi-stage machining system. The following sections will show the derivation of the state space model for multi-stage machining system.

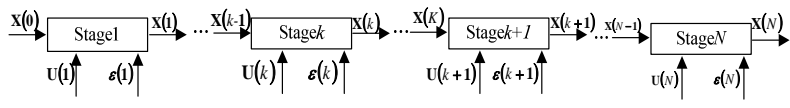

Fig. 1. Diagram of traditional multi station machining process

Actually the position and orientation of coordinate system LCS in coordinate system GCS is determined by fixture parameters. In that situation $\boldsymbol{T}_{\boldsymbol{L}-G}(\boldsymbol{k})$ and $\boldsymbol{R}_{L}^{G}(\boldsymbol{k})$ which transfer the expression of position and orientation of coordinate system LCS into coordinate system GCS are determined by fixture parameters vectors $\boldsymbol{L}^{G}(\boldsymbol{k})$ and can be written as follows

$$
\boldsymbol{T}_{L-G}^{-\mathbf{1}}(k)=\boldsymbol{f}_{\mathbf{1}}\left(\boldsymbol{L}^{G}(\boldsymbol{k})\right) ; \boldsymbol{R}_{L}^{G}(\boldsymbol{k})=\boldsymbol{f}_{\mathbf{2}}\left(\boldsymbol{L}^{G}(\boldsymbol{k})\right)
$$

It should also be noticed that previous machined features denoted as $\boldsymbol{X}^{W}(\boldsymbol{k}-\mathbf{1})$, especially those features used as locating datum, may cause a deviation of workpiece and the attached coordinate system WCS in coor- dinate system LCS in the machining station where operation $\mathrm{k}$ is performed, thus influencing dimensional errors. So $\boldsymbol{X}^{w}(\boldsymbol{k - 1})$ will determine the transformation matrix $\boldsymbol{T}_{W-L}(\boldsymbol{k})$ and the vector $\boldsymbol{R}_{W}^{L}(\boldsymbol{k})$ which describe the orientation and position of coordinate system WCS in coordinate system LCS. Therefore, there exist functions $\boldsymbol{f}_{3}\left(\boldsymbol{X}^{W}(\boldsymbol{k}-\mathbf{1})\right)$ and $\boldsymbol{f}_{4}\left(\boldsymbol{X}^{W}(\boldsymbol{k}-\mathbf{1})\right)$, such that

$$
T_{W-L}^{-1}(k)=f_{3}\left(X^{W}(k-1)\right) ; R_{W}^{L}(k)=f_{4}\left(X^{W}(k-1)\right)
$$

Substituting Eq. (6) and (7) into Eq. (4) gives

$$
\begin{aligned}
\boldsymbol{X}^{W}(\boldsymbol{k})= & \boldsymbol{f}_{\mathbf{3}}\left(\boldsymbol{X}^{W}(\boldsymbol{k}-\mathbf{1})\right) \boldsymbol{f}_{\mathbf{1}}\left(\boldsymbol{L}^{G}(\boldsymbol{k})\right) \boldsymbol{X}^{G}(\boldsymbol{k}) \\
& -\boldsymbol{f}_{3}\left(\boldsymbol{X}^{W}(\boldsymbol{k}-\mathbf{1})\right) \boldsymbol{f}_{\mathbf{1}}\left(\boldsymbol{L}^{G}(\boldsymbol{k})\right) \boldsymbol{f}_{\mathbf{2}}\left(\boldsymbol{L}^{G}(\boldsymbol{k})\right) \\
& -\boldsymbol{f}_{\mathbf{3}}\left(\boldsymbol{X}^{W}(\boldsymbol{k}-\mathbf{1})\right) \boldsymbol{f}_{\mathbf{4}}\left(\boldsymbol{X}^{W}(\boldsymbol{k}-\mathbf{1})\right)
\end{aligned}
$$

Differentiating Eq. (8), finally we have

$$
\Delta \boldsymbol{X}^{W}(\boldsymbol{k})=A(\boldsymbol{k}) \Delta \boldsymbol{X}^{W}(\boldsymbol{k}-\mathbf{1})+\boldsymbol{B}(\boldsymbol{k}) \boldsymbol{U}(\boldsymbol{k})+\boldsymbol{\varepsilon}(\boldsymbol{k})
$$

where

$$
\begin{aligned}
A(\boldsymbol{k})= & \frac{\partial \boldsymbol{f}_{3}}{\partial \boldsymbol{X}(\boldsymbol{k}-\mathbf{1})}\left(\boldsymbol{f}_{1}\left(\boldsymbol{L}^{G}(\boldsymbol{k})\right) \boldsymbol{X}^{G}(\boldsymbol{k})-\boldsymbol{f}_{1}\left(\boldsymbol{L}^{G}(\boldsymbol{k})\right) \boldsymbol{f}_{2}\left(\boldsymbol{L}^{G}(\boldsymbol{k})\right)\right. \\
& -\boldsymbol{f}_{4}\left(\boldsymbol{X}^{\mathrm{W}}(\boldsymbol{k}-\mathbf{1})\right)-\frac{\partial \boldsymbol{f}_{4}}{\partial \boldsymbol{X}(\boldsymbol{k}-\mathbf{1})} \boldsymbol{f}_{3}\left(\boldsymbol{X}^{\mathrm{W}}(\boldsymbol{k}-\mathbf{1})\right) \\
\boldsymbol{B}(\boldsymbol{k}) & =\frac{\partial \boldsymbol{f}_{1}}{\partial \boldsymbol{L}(\boldsymbol{k})}\left(\boldsymbol{f}_{3}\left(\boldsymbol{X}^{w}(\boldsymbol{k}-\mathbf{1})\right) \boldsymbol{X}^{G}(\boldsymbol{k})\right. \\
& \left.-\boldsymbol{f}_{3}\left(\boldsymbol{X}^{\mathrm{w}}(\boldsymbol{k}-\mathbf{1})\right) \boldsymbol{f}_{2}\left(\boldsymbol{L}^{G}(\boldsymbol{k})\right)\right) \\
& -\frac{\partial \boldsymbol{f}_{2}}{\partial \boldsymbol{L}(\boldsymbol{k})} \boldsymbol{f}_{3}\left(\boldsymbol{X}^{w}(\boldsymbol{k}-\mathbf{1})\right) \boldsymbol{f}_{1}\left(\boldsymbol{L}^{G}(\boldsymbol{k})\right) \\
\boldsymbol{U}(\boldsymbol{k})= & \Delta \boldsymbol{L}^{G}(\boldsymbol{k})
\end{aligned}
$$

and vectors $\boldsymbol{\varepsilon}(\boldsymbol{k})$ takes into account the residuals after linearization and un-modeled effects.

Eq. (9) is the needed linear state space model of dimensional variation in multi-station machining systems. $A(\boldsymbol{k})$ is state transition matrix and transfer process deviations $\Delta \boldsymbol{X}^{W}(\boldsymbol{k}-\mathbf{1})$ to state vector $\Delta \boldsymbol{X}^{W}(\boldsymbol{k})$. while $B(\boldsymbol{k})$ is input matrix and combines the incoming part deviation into .

The key issue to establish the Eq. (9) is to find the four expressions:

$$
f_{1}\left(L^{G}(k)\right), f_{2}\left(L^{G}(k)\right), f_{3}\left(X^{W}(k-1)\right), f_{4}\left(X^{W}(k-1)\right),
$$

which were given in[12]. According to the procedures, one can derive the above SOV model for any machining system, provided that adequate CAD/CAPP data about the machining process are available. This makes the process plan evaluation possible prior to the real ma- 
chining process conducted, so that supports the optimum of the process design.

It is noticed that the model only concerns about the influence of fixture parameters errors, previous machined features errors, no other process control characteristics is considered. One can employ the same procedure to establish the state space model concerning more other process control characteristics.

Given the tolerance of the feature machined in this stage is represented as $T(k)$, and then the performance function of the process can be written as:

$$
g(\Delta L(k))=\Delta X^{\mathrm{W}}(k)-\mathrm{T}(k)
$$

\subsection{The Model of Process Reliability}

The calculation of process reliability is to determine the relations of the machined part quality statistical feature and the statistical feature of process character (KCC). The calculation can be fulfilled by the performance function of the process. From the equations (9) and (10), we can easily see that the mathematical function used to describe the variables $\Delta \mathrm{X}(k)$ and $\mathrm{U}(k)$ is nonlinear relation, namely the performance function of the machining process is nonlinear function under the condition that only locator errors are considered. From the theory of reliability, when performance function $\mathrm{g}(\mathrm{x})$ is nonlinear function of variables $\mathrm{X}=\left(\mathrm{X}_{1}, \mathrm{X}_{2}, \cdots, \mathrm{X}_{\mathrm{n}}\right)$, a linearizing work must be done to transfer the nonlinear performance function into linear form, namely $g(\mathrm{X})=a_{0}+\sum_{i=1}^{n} a_{i} \mathrm{X}_{i} \quad$,where $a_{i}(\mathrm{i}=0,1, \cdots, \mathrm{n})$ is constant. As we know, linearzing work is fulfilled by the Taylor series as the following math equation:

$$
\begin{aligned}
\mathrm{Z}= & g\left(\mathrm{X}_{1}, \mathrm{X}_{2}, \cdots, \mathrm{X}_{\mathrm{n}}\right) \\
& \approx g\left(u_{1}, u_{2}, \cdots, u_{n}\right)+\sum_{i=1}^{n}\left(\frac{\partial g}{\partial X_{i}}\right)_{u_{i}}\left(X_{i}-X_{u_{i}}\right)
\end{aligned}
$$

where $u_{X}=u_{X_{1}}, u_{X_{2}}, \cdots, u_{X_{n}}$ is the average point of the performance function, and $\left(\frac{\partial g}{\partial X_{i}}\right)$ is the value of the the differential coefficient function of the performance function at the average point of $u_{X}$.It can be seen that the equation (11) is linear function.

Then mean value $\mu_{g}$ and standard deviation $\sigma_{g}^{2}$ can be written as:

$$
\begin{gathered}
\mu_{g}=g\left(\mu_{X_{1}}, \mu_{X_{2}}, \cdots, \mu_{X_{i}}, \cdots \mu_{X_{n}}\right) \\
\sigma_{g}^{2}=\sum_{i=1}^{n}\left(\frac{\partial g}{\partial X_{i}}\right)_{u_{x}}^{2}+\sum_{i=1}^{n} \sum_{j=1, j \neq i}^{n}\left(\frac{\partial g}{\partial X_{i}}\right)_{u_{X}}\left(\frac{\partial g}{\partial X_{j}}\right)_{u_{x}} \operatorname{Cov}\left(X_{i}, X_{j}\right) \\
=\sum_{i=1}^{n} a_{i}^{2} \sigma_{X_{i}}^{2}+\sum_{i=1}^{n} \sum_{j=1, j \neq i}^{n} a_{i} a_{j} \operatorname{Cov}\left(X_{i}, X_{j}\right)
\end{gathered}
$$

where, $\operatorname{Cov}\left(X_{i}, X_{j}\right)$ is covariance, and $\operatorname{Cov}\left(X_{i}, X_{j}\right)=\rho_{X_{i} X_{j}} \sigma_{X_{i}} \sigma_{X_{j}}, \rho_{X_{i} X_{j}}$ is correlation coefficient of variables $X_{i}$ and $X_{j}$. Wheng(x) is not linear function, we can deal it by linearization.

Since the process variables are independent, so standard deviation $\sigma_{g}^{2}$ can be samplized as:

$$
\sigma_{g}^{2}=\sum_{i=1}^{n} a_{i}^{2} \sigma_{X_{i}}^{2}
$$

According to that the combination of normal distribute variables is also obey the normal distribution, which mean value and standard deviation can be determined by basic variables, the performance function obeys the following normal distribution.

$$
g \sim N\left(\mu_{g}, \sigma_{g}^{2}\right)
$$

The following index $\beta$ can be designed to value the process reliability: 


$$
\beta=\frac{\mu_{\mathrm{g}}}{\sigma_{\mathrm{g}}}=\frac{a_{0}+\sum_{i=1}^{n} a_{i} u_{i}}{\sqrt{\sum_{i=1}^{n} a_{i}^{2} \sigma_{X_{i}}^{2}+\sum_{i=1}^{n} \sum_{j=1, j \neq i}^{n} a_{i} a_{j} \operatorname{Cov}\left(X_{i}, X_{j}\right)}}
$$

So process reliability $P_{r}$ and failure probability $P_{f}$ in first order and second moment (FOSM) can be written as follows:

$$
\begin{aligned}
& P_{r}=P\{g \leq 0\}=P\left\{\frac{g-\mu_{g}}{\sigma_{g}} \leq-\frac{\mu_{g}}{\sigma_{g}}\right\}=\Phi(-\beta) \\
& P_{f}=P\{g>0\}=P\left\{\frac{g-\mu_{g}}{\sigma_{g}}>-\frac{\mu_{g}}{\sigma_{g}}\right\}=\Phi(\beta)
\end{aligned}
$$

where, $\Phi(\bullet)$ is normal standard variable cumulating distribution function.

\subsection{Process Reliability Sensitive Analysis}

Process reliability sensitivity is defined as the derivative of process failure probability $\mathrm{u}_{\mathrm{x}_{\mathrm{i}}}$ to basic variables (including mean value, standard and correlation coefficient). From the relations between index $\beta$ and failure probability $P_{f}$, and between index $\beta$ and basic distribution variables, the process sensitivity can be formulated as follows by means composite deviation:

$$
\begin{gathered}
\frac{\partial P_{f}}{\partial \mu_{X_{i}}}=\frac{\partial P_{f}}{\partial \beta} \frac{\partial \beta}{\partial \mu_{X_{i}}} \\
\frac{\partial P_{f}}{\partial \sigma_{X_{i}}}=\frac{\partial P_{f}}{\partial \beta} \frac{\partial \beta}{\partial \sigma_{X_{i}}} \\
\frac{\partial P_{f}}{\partial \rho_{X_{i} X_{j}}}=\frac{\partial P_{f}}{\partial \beta} \frac{\partial \beta}{\partial \rho_{X_{i} X_{j}}}
\end{gathered}
$$

Because:

$$
P_{f}=\Phi(-\beta)=1-\frac{1}{\sqrt{2 \pi}} \int_{-\infty}^{\beta} \exp \left(-\frac{1}{2} t^{2}\right) d t
$$

So :

$$
\frac{\partial P_{f}}{\partial \beta}=\frac{1}{\sqrt{2 \pi}} \exp \left(-\frac{1}{2} \beta^{2}\right)
$$

and represented as mean value and standard deviation:

$$
\frac{\partial P_{f}}{\partial \beta}=\frac{1}{\sqrt{2 \pi}} \exp \left[-\frac{1}{2}\left(\frac{\mu_{\mathrm{g}}}{\sigma_{g}}\right)^{2}\right]
$$

From the above equations, the locator errors which contribute the product quality fluctuant largely can be diagnosed, and determine the sensitivity of the product quality to locator errors.

Because of the multistage process is highly nonlinear process. The above calculation procedure is very complex, it is very difficult to achieve the process reliability index $\beta$ and following reliability sensitive analysis. Here chaos optimization algorithm and mutative scale chaos optimization algorithm are used to calculate the robust reliability index of non-probability model. By use of the idea of mutative scale, the feasible region is reduced, thereby the searching efficiency is improved and the iteration of optimization is reduced. The detail procedure of the use of chaos optimization algorithm can be seen [13].

\subsection{Summary of the modeling Procedures}

The modeling procedures described in this section can be summarized as follows:

(i) Formula the performance function of the station $\mathrm{k}$, which is the machining feature errors and the caused factors, fixture errors is used in this paper.

(ii) Obtain the distribution feature value $\mu_{\mathrm{g}}$ and $\sigma_{g}$ of the produced KPC at the stage $\mathrm{k}$ by means of the distribution feature value $u_{x_{i}}, \sigma_{x_{i}}$ of the KCC at the stage $\mathrm{K}$.

(iii) Construct an index to formulate the process reliability $P_{r}$ and failure probability $P_{f}$.

(iv) Perform the process reliability sensitive analysis by means of chaos optimization algorithm and mutative scale chaos optimization algorithm .

Figure 2 illustrates the modeling the process reliability for the multiple machined process. 
Note that the above ways to formulate the process reliability model is called the first order and second moment (FOSM)method which will give the approximate value of the process reliability because of the used linearization method. If the more accuracy value is wanted advanced FOSM is desired.

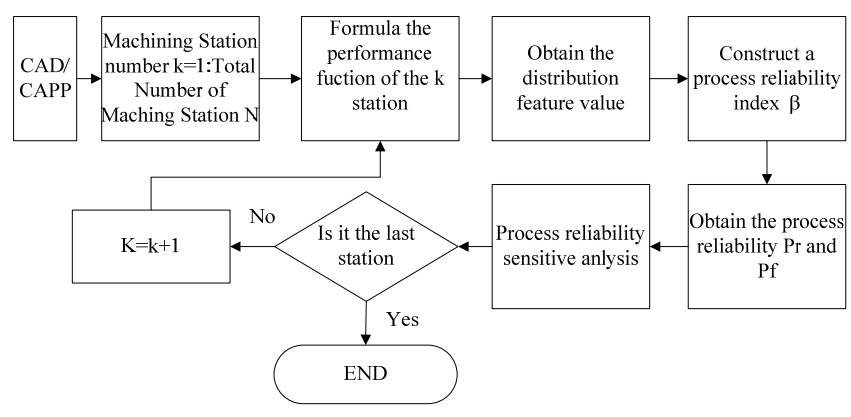

Fig. 2. Flow-chart of the modeling of multistage machining process.

\section{A Simple Two Dimensional Examples}

A simple 2D example is employed to illustrate the procedure outlined in section 2. A rectangular part shown in Fig.3 (a) is machined at the fixture scheme shown in Fig.3 (b). It is assumed that features 1 and 2 have been machined in previous stations and are used as the locating datum at this station. Feature 3 is machined in the station whose locating scheme is shown in Fig.2 (b). Fig.2(c) demonstrates the setup of the workpiece and fixture system. The coordinate systems WCS, LCS and GCS are also shown in Fig.3, respectively.
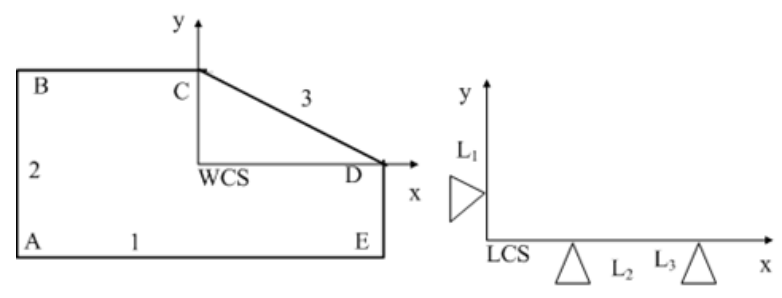

(a) 2D part

(b) Setup scheme

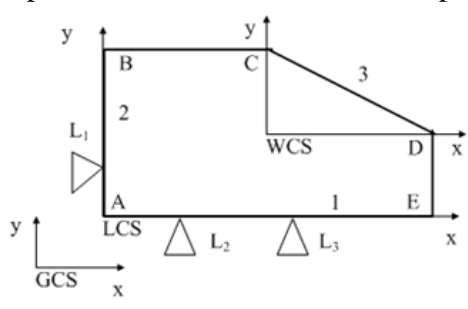

(c) Part in fixture

Fig 3. Simple two-dimensional example
The components of the deviation vector of fixture parameters errors are

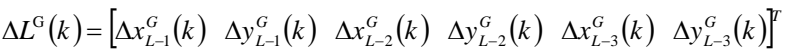

.Assuming

$\Delta x_{L-1}^{G}(k) \Delta y_{L-1}^{G}(k) \Delta x_{L-2}^{G}(k) \Delta y_{L-2}^{G}(k) \Delta x_{L-3}^{G}(k) \Delta y_{L-3}^{G}(k)$

obey $\mathrm{N}(0.02,105), \mathrm{N}(0,1.2) \mathrm{N}(0,2), \mathrm{N}(0,1), \mathrm{N}(0.05,1) \mathrm{N}(0.03,2)$ respectively. By using the equations presented in section 2, the deviation $\Delta X^{\mathrm{w}}(k)$ at the stage is in line with the normal distribution with mean value $\mu_{g(k)}=0.05$, and stand deviation $\sigma_{g(k)}=0.021$,reliability in$\operatorname{dex} \beta=2.38$.So the process reliability sensitivity for the six KPC are calculated as Table 1:

Table 1 . Process reliability sensitivity value

\begin{tabular}{|c|c|c|c|c|c|}
\hline$\partial \mathrm{P}_{\mathrm{t}} / \partial \mu_{\mathrm{x}_{\mathrm{L}-1}}$ & $\partial \mathrm{p}_{\mathrm{f}} / \partial \mu_{\mathrm{y}_{\mathrm{L}-1}}$ & $\partial \mathrm{P}_{\mathrm{f}} / \partial \mu_{\mathrm{x}-2}$ & $\partial \mathrm{P}_{\mathrm{t}} / \partial \mu_{\mathrm{y}_{\mathrm{L}-2}}$ & $\partial \mathrm{p}_{\mathrm{f}} / \partial \mu_{\mathrm{x}_{\mathrm{L}-3}}$ & $\partial \mathrm{P}_{\mathrm{f}} / \partial \mu_{\mathrm{y}_{\mathrm{L}-3}}$ \\
\hline $1.579 \times 10^{-3}$ & $0.05 \times 10^{-3}$ & $0.063 \times 10^{-3}$ & $1.893 \times 10^{-3}$ & $0.035 \times 10^{-3}$ & $2.33 \times 10^{-3}$ \\
\hline$\partial \mathrm{P}_{\mathrm{f}} / \partial \sigma_{\mathrm{X}_{\mathrm{L}-1}}$ & $\partial \mathrm{p}_{\mathrm{t}} / \partial \sigma_{\mathrm{y}_{\mathrm{L}-1}}$ & $\partial \mathrm{P}_{\mathrm{t}} / \partial \sigma_{\mathrm{X}_{\mathrm{L}-2}}$ & $\partial \mathrm{P}_{\mathrm{f}} / \partial \sigma_{\mathrm{y}_{\mathrm{L}-2}}$ & $\partial \mathrm{P}_{\mathrm{f}} / \partial \sigma_{\mathrm{x}_{\mathrm{L}-3}}$ & $\partial \mathrm{P}_{\mathrm{t}} / \partial \sigma_{y_{\mathrm{L}-3}}$ \\
\hline $1.31 \times 10^{-3}$ & $0.03 \times 10^{-3}$ & $0.032 \times 10^{-3}$ & $1.45 \times 10^{-3}$ & $0.01 \times 10^{-3}$ & $1.67 \times 10^{-3}$ \\
\hline
\end{tabular}

From the table, we can see that the contribution of the different process parameters (KPC) to the station part feature quality (KCC) differs. The deviation $\Delta X^{\mathrm{w}}(k)$ of the feature is more sensitive to $\Delta x_{L-1}^{G}(k) \Delta y_{L-2}^{G}(k)$ any $\Delta y_{L-3}^{G}(k)$ than other process parameters, which reveals that we should pay more attention to $\Delta x_{L-1}^{G}(k)$ $\Delta y_{L-2}^{G}(k)$ and $\Delta y_{L-3}^{G}(k)$ when the $\Delta X^{\mathrm{w}}(k)$ controlled.

\section{Conclusion}

Process reliability is one of the important factors which influence the stability of product quality during machining process. How to describe the forming of the process reliability and to calculate it mathematically is an important research Scenario. By means of the state space model of machining process and the performance function, this paper puts forward the method to calculate process reliability. Furthermore, the sensitivity of process reliability is given to facility the diagnosis of the weak points during the multistage machining process. This paper hopes to improve the stability of the machining process. The procedures are essential in the derivation of process reliability and keep the stability of the process. 


\section{Acknowledgment}

Authors want to express their gratefulness to Production Preparation Group of BIT for providing the facilities to this paper. This research is supported by National Department Fundamental Research Foundation(DEDP1901).

\section{References}

1. W.J. Zhang and Y.P. Qian and W. Huang et al., Study on the conception of process reliability in mechanical manufacture, in Reliability, Maintainability and Safety, 2009, ICRMS 2009, pp.995-997.

2. J.K. Jacob and P.S. Sreejith. Process control using reliability based control charts, in Journal of Achievements in Materials and Manufacturing Engineering, 31(2) (2008): 498-504.

3. G. M. Mocko and R Paasch, Incorporating uncertainty in diagnostic analysis of mechanical systems, in Journal of Mechanical Design, Transactions of the ASME, 127 (2) (2005): 315 - 325.

4. Hines JW and Usynin A., Current computational trends in equipment prognostics, in International Journal of Computational Intelligence Systems, 1(1) (2008):94-102.

5. M. Dardalhon and F. Pressecq et al., Evaluation of Process Reliability with Micromechanical Test Structures, in Sensors and Actuators A: Physical, 74(3) (1999):126-133.

6. Alam, Muhammad A., Reliability- and Process-variation aware design of integrated circuits - A broader perspective, in IEEE International Reliability Physics Symposium Proceedings, 2011, 353-363.

7. Ibrahim Habli and Tim Kelly, A Model-Driven Approach to Assuring Process Reliability, in the $19^{\text {th }}$ International Symposium on Software Reliability Engineering, 2008: 1071-1082.

8. LIN G H., Process reliability assessment with a Bayesian approach, in Int J Adv Manuf Technol, 25(2005): 392-395

9. Sun J and Xi L and Pan E., Integration of product quality and tool degradation for reliability modeling and analysis of multi-station manufacturing systems, in International Journal of computer integrated manufacturing, 23(3) (2009): 267-279.

10. J. Jin and Y. Chen, Quality and reliability information integration for design evaluation of fixture system reliability, in Qual. Reliab. Eng. Int, 17(2001):355-372.

11. Y. Chen and J h Jin, Quality-Reliability Chain Modeling for System-Reliability Analysis of Complex Manufactur- ing Processes, in IEEE Transactions on Reliability, 54(3) (2005):475-488.

12. F.P.ZHANG and J.P.LU et al., Locating Error Considered Dimensional Errors Modeling for Multistation Manufacturing System, in Chinese Journal of Mechanical Engineering, 23(6) (2010):765-743.

13. W.Liang. Multi-objective Fuzzy Mutative Scale Chaos Optimization Algorithm and Application, in Proceedings of the 2011 International Conference on Intelligence Science and Information Engineering (ISIE 2011): 93-97 